
allemande

50-1 | 2018

Regards franco-allemands sur la justice dans la construction européenne | Les élections législatives de 2017 en Allemagne et en Autriche

\title{
La réforme du droit allemand de la vente
}

chronique juridique

\section{Sandie Calme}

\section{OpenEdition}

\section{Journals}

Édition électronique

URL : https://journals.openedition.org/allemagne/686

DOI : 10.4000/allemagne.686

ISSN : 2605-7913

Éditeur

Société d'études allemandes

Édition imprimée

Date de publication : 30 juin 2018

Pagination : 207-211

ISSN : 0035-0974

Référence électronique

Sandie Calme, "La réforme du droit allemand de la vente », Revue d'Allemagne et des pays de langue allemande [En ligne], 50-1 | 2018, mis en ligne le 30 juin 2018, consulté le 02 juin 2022. URL : http:// journals.openedition.org/allemagne/686 ; DOI : https://doi.org/10.4000/allemagne.686 


\section{Chronique juridique}

\section{La réforme du droit allemand de la vente}

\section{- Sandie Calme*}

Une réforme substantielle du droit allemand de la vente entre en vigueur au $1^{\text {er }}$ janvier 2018. Elle concerne l'attribution de la charge financière dans le cas où un bien défectueux est intégré à un autre, notamment en matière de construction immobilière.

C'est le cas, par exemple, de la maison construite en y incorporant un matériel défectueux. Qui supporte la responsabilité financière de l'ensemble des dommages occasionnés? Comment organiser, le cas échéant, le partage des responsabilités? Les différents acteurs - dont, notamment, celui ou ceux qui ont fabriqué le produit défectueux, les fournisseurs, vendeurs, acheteurs - peuvent-ils exercer entre eux des droits de recours?

Cette problématique a été mise en valeur au sein de la jurisprudence, et ce jusqu'à celle de la Cour de justice de l'Union européenne. Elle a abouti, en droit allemand, non seulement sur une recherche d'adaptation à la directive européenne, mais encore, au-delà, sur la recherche d'une forme d'équilibre juridique quant à la situation des entrepreneurs.

C'est donc une transition (I) qui s'opère en matière de droit de la vente, à plusieurs niveaux. Elle appelle une réflexion sur l'implication internationale (II) de la réforme.

\section{La transition}

\section{A. L'ancien}

Le droit allemand précédant la réforme qui entre en vigueur le $1^{\text {er }}$ janvier 2018 était porteur d'interrogations sur le sort financier du vendeur qui, ayant livré un bien mobilier défectueux intégré à un autre bien, éventuellement immobilier, par l'acheteur, porterait la charge des réparations et supporterait également l'ensemble des frais

* Docteur en droit, LL.M. (Francfort-sur-le-Main, Allemagne), avocate au barreau de Paris. 
relatifs à la réparation du dommage, y compris le remplacement du bien livré par un bien conforme, et ce sans possibilité de recours contre le fournisseur dans le cas où ce vendeur est un entrepreneur.

Initialement, l'article $\$ 433$ du Code civil allemand (Bürgerliches Gesetzbuch, BGB) dispose, en son alinéa $1^{\text {er }}$, que "par le contrat de vente, le vendeur d'un bien est tenu d'en effectuer la délivrance à l'acheteur et de lui en transmettre la propriété» et que «le vendeur est tenu de transmettre à l'acheteur le bien exempt de défaut matériel ou juridique».

Au sein de l'article $\$ 434$ du Code civil allemand, il est précisé que «le bien est exempt de défaut matériel si, lors du transfert des risques, il présente les qualités convenues ».

L'article $\$ 437$ du Code civil allemand offre des options à l'acheteur du bien vicié: demande d'exécution ultérieure, résolution du contrat, réduction du prix de vente, indemnisation ou remboursement des frais engagés.

Selon l'ancien article $\$ 439$ du Code civil allemand:

«1. L'acheteur peut à son gré demander, à titre d'exécution a posteriori, soit la suppression du défaut, soit la livraison d'un bien exempt de défaut.

2. Le vendeur doit supporter les dépenses nécessaires à l'exécution a posteriori, notamment le coût de transport, d'acheminement, de main-d'œuvre et des matériaux.

3. Le vendeur [...] peut refuser le mode d'exécution a posteriori choisi si celui-ci entraîne obligatoirement des coûts disproportionnés. Il faut en particulier considérer la valeur du bien exempt de défaut, l'importance du défaut et la question de savoir si l'autre mode d'exécution a posteriori peut être préféré sans grand inconvénient pour l'acheteur. Le droit de l'acheteur se limite dans ce cas à l'autre mode d'exécution a posteriori; le droit du vendeur de refuser également celui-ci dans les conditions de la première phrase reste applicable.

4. Si le vendeur livre un bien exempt de défaut en vue de l'exécution a posteriori, il peut demander de l'acheteur la restitution du bien défectueux [...]».

\section{B. Le nouveau}

Avec la nouvelle réforme, l'entrepreneur, en sa qualité de vendeur du bien vicié, peut désormais exercer un recours contre le fournisseur (article 445a du Code civil allemand) du bien défectueux vendu, afin que ce fournisseur soit amené à assumer, le cas échéant, les frais occasionnés par le vice de la chose incorporée. Cela n'est pas valable pour les biens d'occasion et le vice doit être présent avant le transfert de la charge des risques. Cela va de soi dans la mesure où le fournisseur est tenu de livrer un bien non vicié ou bien, dans le cas du bien d'occasion, un bien dont les caractéristiques sont connues et assumées par son acheteur. Le recours du vendeur contre le fournisseur s'étend à l'ensemble des fournisseurs subséquents avec la même exigence au niveau du temps du transfert de la charge des risques. Des spécificités s'appliquent au niveau des délais.

Le nouvel article $\$ 439$ alinéa 3, première phrase, du Code civil allemand, dispose que le vendeur est tenu, dans le cadre de l'exécution ultérieure, de compenser à l'acheteur les dépenses nécessaires à l'extraction du bien vicié ainsi que les frais d'insertion du bien réparé ou du bien de substitution indemne, si l'acheteur a inséré ou associé le bien vicié à un autre selon sa nature et conformément à l'usage auquel il est destiné, que le vendeur et l'acheteur aient ou non la qualité d'entrepreneurs.

L'acheteur consommateur est au bénéfice du droit d'exiger une provision, selon le nouvel article $\$ 475$ alinéa 6 du Code civil allemand. 
L'acheteur consommateur n'a toutefois pas droit à indemnisation pour ses efforts de réparation s'il connaissait le vice du bien au moment de son insertion ou association à l'autre bien (articles $\$ 439$ alinéa 3, seconde phrase et $\$ 442$ alinéa $1^{\text {er }}$ du Code civil allemand).

Dans le cas de l'acheteur consommateur, le vendeur peut limiter l'indemnité à un montant modéré si l'amélioration ou la substitution du bien vicié supposent des frais élevés de manière disproportionnée, eu égard, en particulier, à la valeur du bien vendu à l'état indemne et à la consistance du vice (article $\$ 475$ du Code civil allemand).

À l'échelle transnationale, la configuration du bien inséré ou associé à un autre pose la question de la loi applicable et de l'implication des directives dans le cadre du droit de l'Union européenne.

\section{L'implication internationale de la réforme}

\section{A. Détermination de la loi applicable}

En matière de droit des biens, la norme de référence, présentant un certain caractère d'ordre public international, est la lex rei sitae, à savoir la loi du lieu de localisation du bien en cause, immobilier comme mobilier. En matière de biens meubles, cette règle est appelée à suivre le bien en quelque lieu qu'il se trouve. Lapplication de la règle est particulièrement ancrée en matière immobilière, par exemple selon l'article $3 \mathrm{du}$ Code civil français. Si un bien immobilier est localisé en France, le bien meuble vicié qui y sera incorporé deviendra un immeuble par destination et verra son sort régi par le droit français. Il en sera de même pour le bien immobilier situé, par exemple, en Allemagne, ou pour les biens meubles insérés les uns dans les autres et qui se déplacent au-delà de frontières.

\section{B. Source de droit de l'Union européenne}

La Cour de justice de l'Union européenne a jugé, en date du 16 juin 2011 (affaire $n^{\circ}$ C65/09), que «l'article 3, paragraphes 2 et 3, de la directive 1999/44/CE du Parlement européen et du Conseil, du 25 mai 1999, sur certains aspects de la vente et des garanties des biens de consommation, doit être interprété en ce sens que, lorsqu'un bien de consommation non conforme, qui, avant l'apparition du défaut, a été installé, de bonne foi, par le consommateur conformément à sa nature et à l'usage recherché, est mis dans un état conforme par remplacement, le vendeur est tenu soit de procéder lui-même à l'enlèvement de ce bien du lieu où il a été installé et d'y installer le bien de remplacement, soit de supporter les frais nécessaires à cet enlèvement et à l'installation du bien de remplacement», peu important si le vendeur «s'était engagé, en vertu du contrat de vente, à installer le bien de consommation acheté initialement».

Elle a précisé que «l'article 3, paragraphe 3, de la directive 1999/44» interdit «qu’une législation nationale accorde au vendeur le droit de refuser le remplacement d'un bien non conforme, seul mode de dédommagement possible, au motif que celui-ci lui impose, en raison de l'obligation de procéder à l'enlèvement de ce bien du lieu où il a été installé et d'y installer le bien de remplacement, des coûts disproportionnés au regard de la valeur qu'aurait le bien s'il était conforme et de l'importance du défaut de conformité », tout en autorisant la limitation du «droit du consommateur au 
remboursement des frais d'enlèvement du bien défectueux et d'installation du bien de remplacement» «à la prise en charge, par le vendeur, d'un montant proportionné».

\section{Parallèle en droit français}

En droit français, c'est l'ordonnance no 2005-136 du 17 février 2005 relative à la garantie de la conformité du bien au contrat due par le vendeur au consommateur qui transpose ces dispositions du droit de la consommation, modifiant ainsi notamment le Code civil et le Code de la consommation.

La section 2 de l'ordonnance prévoit une garantie légale de conformité. Ainsi, dans notre cas de figure, le vendeur (article L.211-4 du Code de la consommation) répond des défauts de conformité résultant de l'emballage, des instructions de montage ou de l'installation lorsque celle-ci a été mise à sa charge par le contrat ou a été réalisée sous sa responsabilité.

Pour être conforme au contrat (article L. 211-5), le bien doit:

- être propre à l'usage habituellement attendu d'un bien semblable et, le cas échéant, correspondre à la description donnée par le vendeur et posséder les qualités que celui-ci a présentées à l'acheteur sous forme d'échantillon ou de modèle;

- présenter les qualités qu'un acheteur peut légitimement attendre eu égard aux déclarations publiques faites par le vendeur, par le producteur ou par son représentant, notamment dans la publicité ou l'étiquetage;

- ou présenter les caractéristiques définies d'un commun accord par les parties ou être propre à tout usage spécial recherché par l’acheteur, porté à la connaissance du vendeur et que ce dernier a accepté.

Les défauts de conformité qui apparaissent dans un délai de six mois à partir de la délivrance du bien sont présumés exister au moment de la délivrance, sauf preuve contraire, et le vendeur peut combattre cette présomption si celle-ci n’est pas compatible avec la nature du bien ou le défaut de conformité invoqué (article L. 211-7).

L'acheteur est en droit d'exiger (article L.211-8) la conformité du bien au contrat. Il ne peut cependant contester la conformité en invoquant un défaut qu'il connaissait ou ne pouvait ignorer lorsqu'il a contracté. Il en va de même lorsque le défaut a son origine dans les matériaux qu'il a lui-même fournis.

En cas de défaut de conformité, l'acheteur choisit (article L. 211-9) entre la réparation et le remplacement du bien. Le vendeur peut ne pas procéder selon le choix de l'acheteur si ce choix entraîne un coût manifestement disproportionné au regard de l'autre modalité, compte tenu de la valeur du bien ou de l'importance du défaut. Il est alors tenu de procéder, sauf impossibilité, selon la modalité non choisie par l'acheteur.

Si la réparation et le remplacement du bien sont impossibles (L.211-10), l'acheteur peut rendre le bien et se faire restituer le prix ou garder le bien et se faire rendre une partie du prix. La même faculté lui est ouverte si la solution demandée, proposée ou convenue en application de l'article L.211-9 ne peut être mise en œuvre dans le délai d'un mois suivant la réclamation de l'acheteur, ou si cette solution ne peut l'être sans inconvénient majeur pour celui-ci compte tenu de la nature du bien et de l'usage qu'il recherche. La résolution de la vente ne peut toutefois être prononcée si le défaut de conformité est mineur. 
L'application des dispositions des articles L.211-9 et L.211-10 a lieu sans aucun frais pour l'acheteur, et ces mêmes dispositions ne font pas obstacle à l'allocation de dommages et intérêts (article L.211-11).

L'action récursoire (article L.211-14) peut être exercée par le vendeur final à l'encontre des vendeurs ou intermédiaires successifs et du producteur du bien meuble corporel, selon les principes du Code civil. On entre dans le cadre de la responsabilité du fait des produits défectueux (articles 1245 et suivants du Code civil).

L'ordonnance encadre aussi un système de garantie non pas légale mais commerciale. L'ordonnance de 2005 ajoute un élément d'ordre public international par lequel la protection accordée par un État membre de l'Union européenne qui transpose la directive suit le justiciable:

«Quelle que soit la loi applicable au contrat, l'acheteur qui a sa résidence habituelle dans un État membre de la Communauté européenne ne peut être privé de la protection que lui assurent les dispositions prises par cet État en application de la directive 1999/44/CE du Parlement européen et du Conseil du 25 mai 1999 et qui ont un caractère impératif:

- si le contrat a été conclu dans l'État du lieu de résidence habituelle de l'acheteur;

- ou si le contrat a été précédé dans cet État d'une offre spécialement faite ou d'une publicité et des actes accomplis par l'acheteur nécessaires à la conclusion dudit contrat;

- ou si le contrat a été conclu dans un État où l'acheteur s'est rendu à la suite d'une proposition de voyage ou de séjour faite, directement ou indirectement, par le vendeur pour l'inciter à contracter» (article L. 211-8).

Comme le droit français, le droit allemand va au-delà du cadre posé par le droit de l'Union européenne tel qu'interprété par la Cour de justice de l'Union européenne. $\mathrm{Au}$ regard de la question de la loi applicable et de la préservation de l'ordre public international, le droit français de la vente prévoit également des cas d'application par ses juridictions du droit d'autres États membres de l'Union européenne, par exemple du droit allemand. 